\title{
Aristolochic acid-one of the most potent carcinogens known to man
}

\begin{abstract}
Although aristolochic acid-a compound found in the Aristolochiaceae family of plants, including wild ginger-is known to cause kidney damage and cancers of the urinary tract and has been banned in Europe, Asia, and North America, this plant product is still commonly used in Chinese herbal remedies for slimming, menstrual symptoms, and rheumatism.
\end{abstract}

Now, two studies co-published in Science Translational Medicine have shown that mutations caused by aristolochic acid form a unique molecular signature that can be used as a 'fingerprint' to identify aristolochic acid as the cause of cancer. This molecular signature is characterized by a predominance of A:T-to-T:A transversions - a relatively unusual type of mutation that is not generally seen in cancers caused by other carcinogenswhich are concentrated at splice sites, resulting in the insertion or deletion of whole exons of mRNA.

In the first study, Poon et al. performed whole-genome and exome analysis of DNA from nine patients with upper urinary tract urothelial cell carcinoma (UTUC) and known aristolochic acid exposure. They observed a high frequency of somatic mutations in chromatin modifiers, particularly KDM6A, and reproduced the aristolochic acid molecular signature by treating human renal tubular cells with this plant product. In addition, they screened the genomes and exomes of 93 patients with hepatocellular carcinomas and identified very similar mutational signatures in 11 of them, suggesting that aristolochic acid (or a similar compound) also has a carcinogenic role in a subset of liver cancers.

"As far as we know, this is a novel application of the use of a carcinogen's molecular signature to detect its involvement," says Steve Rosen, one of the lead authors of the study by Poon and co-workers. "Obviously, we can potentially use a similar approach to identify the molecular fingerprints of other carcinogens and, therefore, detect their involvement in other cancers or diseases."

In the second study, Hoang and colleagues performed exome sequencing for 26 individuals with UTUC (19 with known aristolochic acid exposure and seven without) and identified the same molecular fingerprint. However, these study authors are less confident that mutational signatures can be identified for other carcinogens, allowing us to pinpoint the exact cause of each patient's cancer. "This approach is probably only appropriate for the subset of carcinogens that have a mutational profile sufficiently distinct from the type of mutations produced in the course of normal metabolism," explains Tom Rosenquist, who, along with Kenneth Kinzler, led the research performed by Hoang et al.

Regardless, the authors of both studies are in agreement regarding the immediate implications of this work, namely the importance of limiting aristolochic acid availability and screening patients who

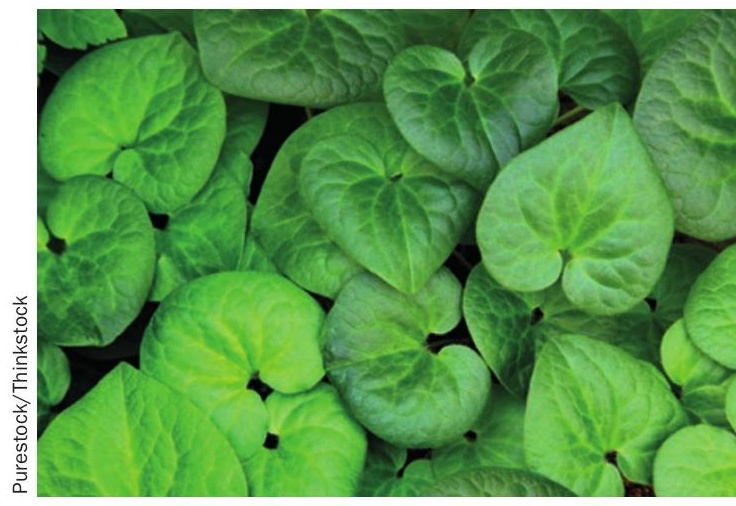

have been exposed to this carcinogen. Alarmingly, the average mutation rate of 150 mutations/Mb in patients with aristolochic-acid-associated UTUC is substantially greater than previously reported rates for smoking-associated lung cancer and UV-associated melanoma (8 mutations/Mb and 111 mutations/Mb, respectively), making it one of the most potent known carcinogens on the planet.

"The high prevalence of aristolochicacid-induced UTUC in Taiwan has been well documented, but prevalence in other Asian countries is unknown," says Rosenquist. "Our work opens the possibility of detecting the mutated DNA in plasma or urine from patients at risk and provides a tool for documenting aristolochic acid involvement in these populations."

Melanie Clyne

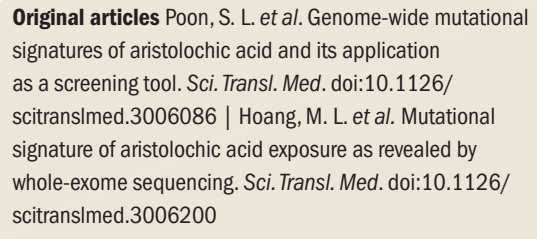

